\title{
The methods and automatic technology aimed at imagery georeferencing, cloud screening, atmospheric and radiometric correction of KMSS-M satellite data
}

\author{
Pavel Kolbudaev ${ }^{1}$, Dmitry Plotnikov ${ }^{1 *}$, Evgeny Loupian ${ }^{1}$, Andrey Proshin ${ }^{1}$ and Alexey \\ Matveev ${ }^{1}$ \\ ${ }^{1}$ Space Research Institute of the Russian Academy of Sciences, 117997 Moscow, Russia
}

\begin{abstract}
In this study we present methods and automatic technology developed for routine processing of satellite imagery acquired by cameras MSU-201 and MSU-202 (KMSS-M) onboard Meteor-M №2. The developed methods were aimed at imagery georeferencing issues fixing, clouds and shadows detection as well as atmospheric and radiometric correction. Basing on these methods we built an automatic technology and complete KMSS-M data processing chain which provided analysis ready dataset for Russian grain belt and adjacent areas of neighboring countries for the year 2020. Method for imagery georeferencing was based on Pearson's correlation localized maximization when compared to the georefenced and cloudfree coarse-resolution reference image produced in IKI RAS through MOD09 product time series processing. Method for clouds and shadows detection was based both on the spatial analysis of outputs from geocorrection step and auxiliary image, characterizing georeferenced KMSS-M image values relative accordance with the IKI reference image. The atmospheric correction was based on localized histogram matching of KMSS-M and IKI reference date-corresponding imagery, and thereby concurrently performed radiometric correction of KMSS-M data, compensating effects of varying viewing and illumination geometry which explicitly manifest across $960-\mathrm{km}$-wide swath area. The developed methods are noticeably minimalistic, requiring only one target spectral band to perform properly. Due to high flexibility and robustness, they also may be applied to raw satellite imagery acquired from various Earth observation systems, including Russian systems of high and moderate spatial resolution. The technology is currently being deployed in an operative mode for several test sites of Russia since the year 2021 onwards.
\end{abstract}

Meteor-M No. 2 meteorological satellite, which carries KMSS-M instrument (acronym for multispectral satellite imagery complex), was launched on July 8, 2014 and placed on sunsynchronous orbit at an altitude of about $825 \mathrm{~km}$ [1]. The KMSS-M consists of two cameras, designated MSU-201, MSU-202, and one additional camera, designated MSU-

\footnotetext{
* Corresponding author: $\underline{\text { dmitplot } @ \text { d902.iki.rssi.ru }}$
} 
250. In this study we focus on data acquired by two multispectral cameras MSU-201(2). The cameras are optimized for land monitoring and operate in three spectral bands - green (0.535-0.575 microns), red (0.630-0.680 microns) and near infrared (0.760-0.900 microns) with a spatial resolution of about 60 meters, the formed viewing band from two cameras is about $960 \mathrm{~km}$ [1]. The CCD elements of the corresponding spectral channels are separated in the focal plane in the direction of satellite's orbital motion, which leads to interband parallax, evident on elevated objects such as clouds, mountain peaks, as well as to a time lag between recording one objects' reflectance in different spectral bands. KMSS-M imagery is characterized by a rare combination of spatial resolution and acquisition repeatability, so Russia is completely covered with KMSS-M imagery every 2-3 days. The launch of the Meteor-M satellite No. 2-2 on July 5, 2019 with the following KMSS device onboard provides full coverage of Russia with virtually daily acquisitions.

The developed methods of georeferencing, cloud and shadow screening, as well as radiometric and atmospheric correction are based on spatial analysis and localized comparison with a date-corresponding coarse-resolution reference image, produced in IKI RAS using reconstruction methods $[2,3]$. The reference image contains measurements of surface reflectance reconstructed data for required date and is free from hindering factors (clouds, shadows, snow cover). MODIS reference images were obtained by interpolating a time series of MOD09 product using LOESS weighted interpolation approach [2], where normalized values of the NDSI index, sensitive to the influence of interfering factors, were used as weight factors. With Sentinel-2 (MSI) high resolution imagery archive for entire territory of Russia for the year 2020, it was found that reconstructed reference is better geolocated compared to the original MOD09 product, featuring average geolocation error of about 15 meters (about $6 \%$ of the pixel size).
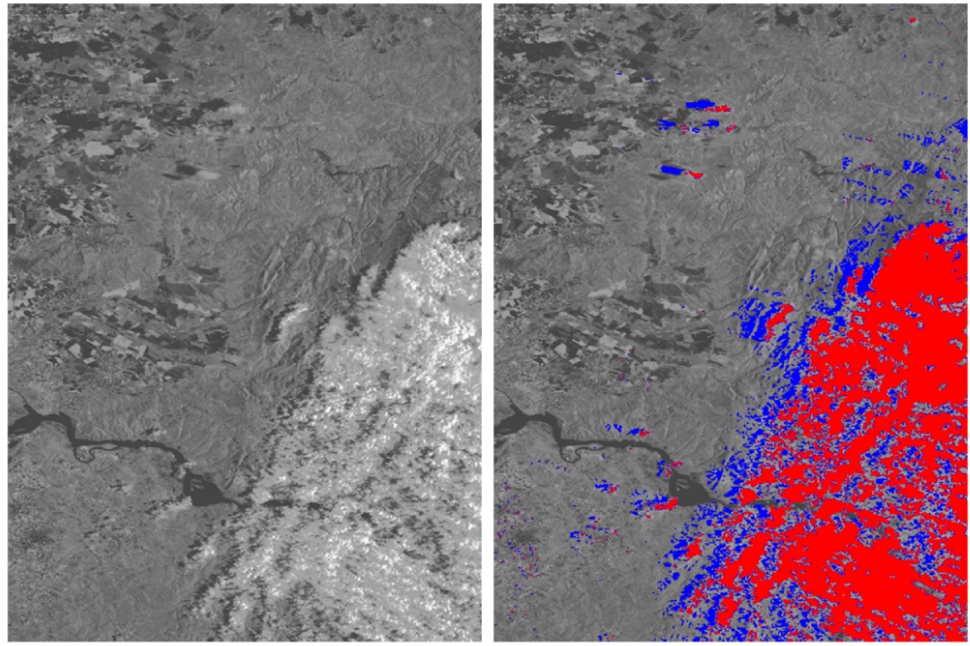

Fig. 1. Part of clouded MSU-201 scene (NIR band) for May 15, 2020. Detected clouds are marked red, shadows are marked blue

Two-staged approach for georeferencing of KMSS-M data is based on an optimized search of possible positions of the MSU-201(2) image relative to the reference image and finding an spatial offset at which the value of Pearson's correlation between data is maximized. At the first stage the systematic geolocation bias of the MSU-201(2) image is eliminated through Pearson's correlation maximization using the whole scene data. At the 
second stage geocorrection process operates with small local areas (blocks) of the original image. When calculating correlation, the portions of the KMSS-M images are sequentially coarsed in a sliding window of 4 by 4 pixels and the result of coarsening is compared with the corresponding pixel of MODIS reference low-resolution image. The search for the optimal position of the block is performed with a spatial stepping equal to one KMSS-M pixel. The distinctive feature of this method is the ability to geocorrect cloudless areas of high spatial resolution images using low spatial resolution reference in separate spectral bands independently. The comparison of geo-corrected KMSS-M images with the collection of cloudless Sentinel-2 (MSI) images for entire territory of Russia for the 2020 year indicates that average geolocation error does not exceed 40 meters.

The cloud detection method is based on the use of the maximum values of Pearson's correlation, stored as an image, obtained at the stage of georeferencing and which characterizes the presence and degree of surface hindering factors. Such image is thresholdclassified into two classes - mostly clear and mostly clouded areas. Comparison of the KMSS-M image with MODIS reference and the use of the described auxiliary binary classification enable to set a local adaptive local threshold for cloud detection and thus calculate cloud masks.

The shadow masking method is based on Sun illumination geometry reconstructed using geographic coordinates and image acquisition timestamps. The area of potential cloud shadows is determined using calculated scene illumination geometry and auxiliary image of the difference between KMSS-M and the reference image. Figure 1 shows an example of highlighting clouds and shadows on a part of the MSU-201 for May 15, 2020. The advantage of the method is the ability to detect clouds and shadows using raw and uncalibrated data in separate spectral bands independently.

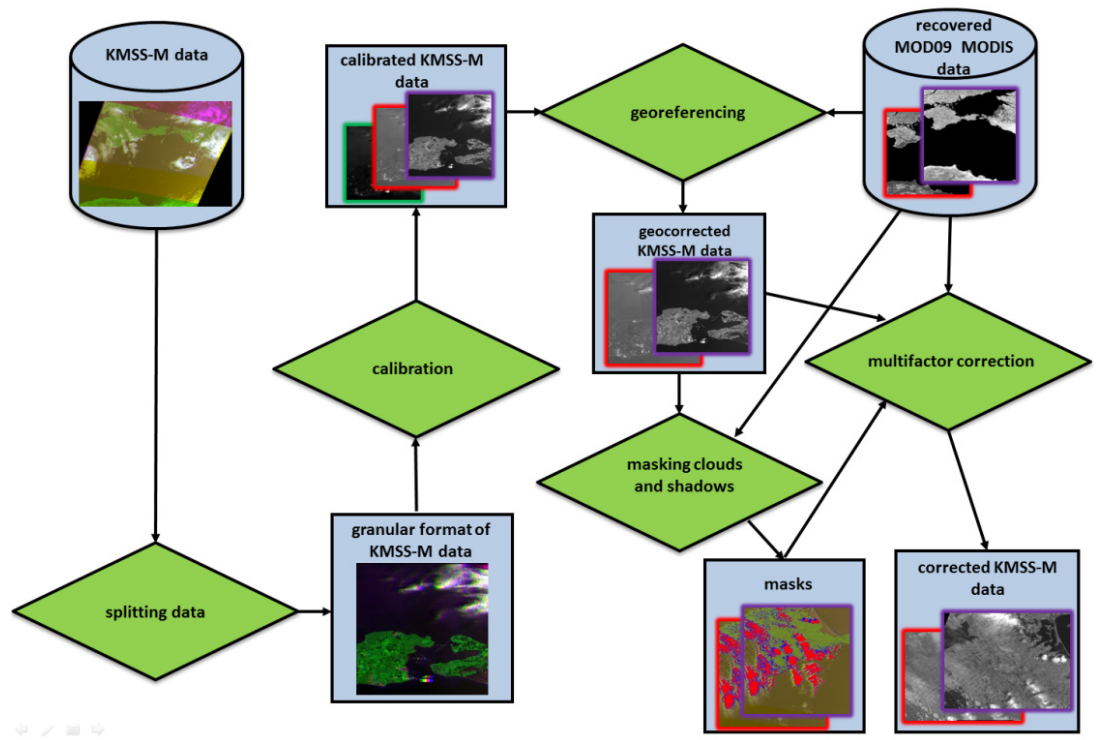

Fig. 2. General flowchart of KMSS-M data processing technology 
The method for radiometric and atmospheric correction of KMSS-M images is based on localized and joint spatial analysis of cloudless image areas with the MODIS data. In the process of joint spatial analysis a variable-size sliding window collects local statistics of the required volume characterizing the localized distribution of brightness values in the compared data sources. Because of the area of analysis, date of the reference and the KMSS-M fit the resulting distributions both histograms have the same form and can be match to each other on the assumption that the influence of the atmosphere shifts and deforms the histograms of the brightness distribution [4]. For each local sliding window centered in the local area of the image (block) linear transformation coefficients are automatically calculated and applied to the pixels of window. As a result each pixel of the image being corrected and receives many estimates from its own and surrounding blocks with level of accuracy of estimates. The combination of all estimates makes it possible to convert the initial values on the KMSS-M to the values of the spectral brightness of the reference image.

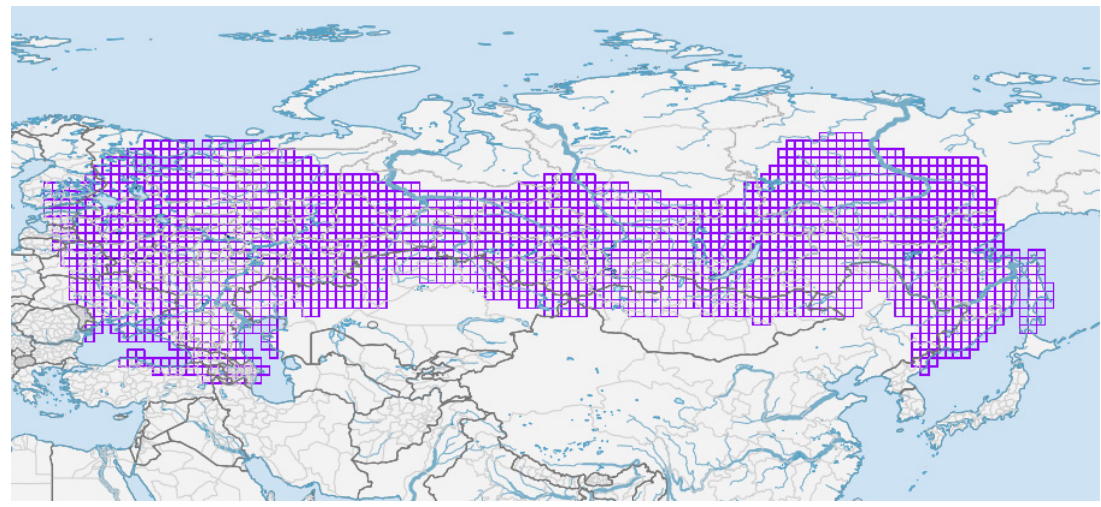

Fig. 3. Availability map for KMSS-M standard products acquired during the year 2020 with Eurasian continent on background

The method of radiometric calibration and atmospheric correction of KMSS-M images suppresses radiometric effects which appear due to a significant difference in observation and illumination angles across $960 \mathrm{~km}$ of swath. The advantage of the described method of multifactor correction is the ability to process each spectral band independently.

The developed methods of georeferencing, clouds and shadows detection, as well as radiometric and atmospheric correction were used as the basis for the automatic technology for routine data processing of KMSS-M imagery deployed at the IKI RAS [5]. The technology implements spatial re-arrangement of the data from scenes to a granular form together with more convenient naming for all derived products that are more convenient for their spatial-temporal analysis, cataloging and distribution. In the approved standard the file names include information about the product name, date (year, day and time), sensor ID and granule number. The flowchart of the developed technology is shown in Fig. 2. The results of the technology are available in the cartographic interface of the Sozvezdie-Vega family systems (sozvezdie-vega.ru) and include a standard set of products: atmospherically corrected and georeferenced KMSS-M images (Red and NIR bands), cloud 
and shadow masks for each spectral channel separately as well as NDVI [8]. These products are processed for the areas of Russian grain belt and neighboring countries and available for download and analysis. The data processing is carried out using the resources of the collective use center «IKI-Monitoring" [6].

\section{References}

1. B. Zhukov, T. Kondratieva, I. Polyanskiy, Interannual sensitivity trend of the cameras of the multispectral satellite imaging system KMSS-M on Meteor-M No. 2 spacecraft based on the in-flight calibration in 2015-2020, Sovremennye problemy distantsionnogo zondirovaniya Zemli iz kosmosa, Vol. 18, No. 1, pp. 53-60 (2021)

2. W. Cleveland, Robust Locally Weighted Regression and Smoothing Scatterplots, American Statistical Association, V. 74, 368, P. 829-836 (1979). DOI: $10.2307 / 2286407$.

3. D. Plotnikov, E. Elkina, E. Dunaeva, S. Khvostikov, E. Loupian, S. Bartalev, Development of the Method for Automatic Winter Crops Mapping by Means of Remote sensing Aimed at Crops State Assessment over the Republic of Cremia, Taurida Herald of the Agrarian Sciences, No. 1, 21, P. 64-83 (2020). DOI 10.33952/2542-0720-20201-21-64-83. 7.

4. D. Hadjimitsis, G. Papadavid, A. Agapiou, K. Themistocleous, M. Hadjimitsis, A. Retalis, S. Michaelides, N. Chrysoulakis, L. Toulios, C. Clayton, Atmospheric correction for satellite remotely sensed data intended for agricultural applications: impact on vegetation indices, Natural Hazards and Earth System Sciences, V. 10, P. 89-95 (2010)

5. D. Plotnikov, P. Kolbudaev, B. Zhukov, A. Matveev, S. Bartalev, V. Egorov, A. Kashnitskiy, A. Proshin, The collection of multispectral KMSS-M (Meteor-M No. 2) satellite data aimed at quantitative assessment of the Earth surface, Sovremennye problemy distantsionnogo zondirovaniya Zemli iz kosmosa, Vol. 17, No. 7, P. 256-262 (2020). DOI: 10.21046/2070-7401-2020-17-7-256-262

6. E. Loupian, A. Proshin, M. Bourtsev, A. Kashnitskiy., I. Balashov, S. Bartalev, A, Konstantinova, D. Kobets, A. Mazurov, V. Marchenkov, A. Matveev, M. Radchenko, I. Sychugov, V. Tolpin, I. Uvarov, Experience of development and operation of the IKI-Monitoring center for collective use of systems for archiving, processing and analyzing satellite data, Sovremennye problemy distantsionnogo zondirovaniya Zemli iz kosmosa, Vol. 16, No. 3, P. 151-170 (2019). DOI: 10.21046/2070-7401-2019-16-3151-170 